\title{
Perforant Path Activation Modulates the Induction of Long-Term Potentiation of the Schaffer Collateral-Hippocampal CA1 Response: Theoretical and Experimental Analyses
}

\author{
William B. Levy, ${ }^{1,2,3}$ Nancy L. Desmond, ${ }^{1}$ and De Xing Zhang ${ }^{1}$ \\ Departments of ${ }^{1}$ Neurological Surgery and ${ }^{2}$ Psychology \\ University of Virginia \\ Charlottesville, Virginia 22908 USA
}

\begin{abstract}
In one computational model of hippocampal function, the entorhinal cortical input to CA1 is hypothesized to play a key role in the ability of CA1 to decode CA3 recodings. Here, we develop a modification of this CA1 decoder hypothesis that is applicable to several computational theories of hippocampal function, and then we electrophysiologically investigate one assumption of this new hypothesis. First, using biologically realistic estimates, we calculate that CA3-induced CA1 excitation is too high and that inhibition plausibly plays a role in this CA1 decoder model. Thus motivated, we turn to a physiological demonstration to substantiate the plausibility of the proposed mechanism. Using the rat hippocampal slice, we examine an interlaminar interaction between the distal perforant path input to hippocampal CA1 stratum moleculare and the more proximal Schaffer collateral input to stratum radiatum. Perforant path activation provides sufficient inhibition to block homosynaptic long-term potentiation elicited by a suitably strong stratum radiatum input. For this interlaminar interaction to be most effective, perforant path activation must both precede and follow Schaffer collateral activation. Perforant path-evoked inhibition in CA1 can
\end{abstract}

${ }^{3}$ Corresponding author. thus serve as a viable mechanism in the learned decoder theory of hippocampal CA1.

\section{Introduction}

In some theories of hippocampal function, the CA1 region is viewed as a decoder of CA3 recodings (Levy 1989; Rolls 1989; Burgess et al. 1994; O'Reilly and McClelland 1994; Hasselmo 1995). One such theory (Levy 1989) postulates a strong, excitatory entorhinal cortical (EC) input to CA1 stratum moleculare that (1) forces a CA1 neuron to fire whenever the appropriate EC inputs are active and (2) provides the postsynaptic excitation needed to associatively potentiate a converging, coactive CA3-CA1 synapse in stratum radiatum.

Several experimental reports, however, cast doubt on this model. In particular, perforant path activation apparently engages a large inhibitory response in the CA1 pyramids (Lambert et al. 1991; Colbert and Levy 1992; Empson and Heinemann 1995; Soltesz 1995). Although some EC afferents to CA1 are excitatory (Colbert and Levy 1992; Empson and Heinemann 1995), they are highly topographic and are easily overwhelmed by the dispersed and largely disynaptically activated inhibition.

As a result of these experimental observations, the original model of a CA1 decoder (Levy et al. 1990) must be discarded (Levy et al. 1995) or, at the very least, significantly modified. One modification (Hasselmo and Schnell 1994) posits that the strength of the stratum moleculare input varies depending on cholinergic activation of CA1. Here, we develop another idea.

LEARNING \& MEMORY 4:510-518 @ 1998 by Cold Spring Harbor Laboratory Press ISSN1072-0502/98 \$5.00

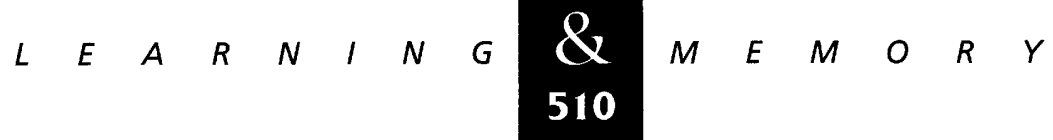


We take advantage of the fact that Schaffer collateral-CA1 synapses are numerous and excitatory but that their activity is sparse when considered on the time scale of neuronal integration. That is, these CA3 efferents only moderately excite CA1. At this moderate level of excitation, a small subpopulation of the CA1 pyramids is always near threshold for associative long-term potentiation (LTP) (see Fig. 1A). Some cells are depolarized slightly beyond the threshold for LTP, whereas others remain hyperpolarized relative to this threshold.

In our revised hypothesis that we evaluate below, the EC input to CA1 has two different functions. First, the topographically precise, excitatory EC input excites a small number of CA1 pyramidal cells that are already depolarized near to or beyond threshold by their CA3 input (as in Fig. 1A). Second, and at the same time, the widely distributed, net inhibition evoked by the EC input prevents any CA1 pyramids that are beyond threshold, but not activated by perforant path excitation, from undergoing LTP (as in Fig. 1B). That is, a set of CA1 pyramidal cells receives excitatory drive from $\mathrm{CA} 3$ that puts them near or beyond the threshold for LTP induction at the CA3-CA1 synapses. This moderate excitation by CA3 thereby allows the perforant path input to select, in both a positive and a negative fashion, which CA1 neurons will associatively potentiate their CA3-CA1 synapses. Thus, as a function of both its inhibitory and its excitatory influences, the perforant path input can "sort" CA1 pyramidal cells by moving their level of excitation above or below the threshold for LTP induction.

For this hypothesis to be viable, a suitable number of CA1 cells must straddle the threshold for cell discharge, and the inhibition generated by inputs to stratum moleculare must be able both to permit and to prevent LTP. The viability of the first assumption is assessed analytically in Results. Two experimental tests are also required to demonstrate the feasibility of this new CA1 decoder hypothesis. First, the perforant path input to CA1 must be able to drive subthreshold pyramidal cells either to threshold or beyond (Fig. 1A). Indeed, such cooperative interactions can produce the permissive postsynaptic input that allows LTP of coactive Schaffer collateral inputs (Levy et al. 1995). Second, perforant path activation must be able to hyperpolarize, to below threshold, those pyramidal cells that are depolarized above threshold by their Schaffer collateral inputs (Fig. 1B). Here we address this second test by asking whether activa-
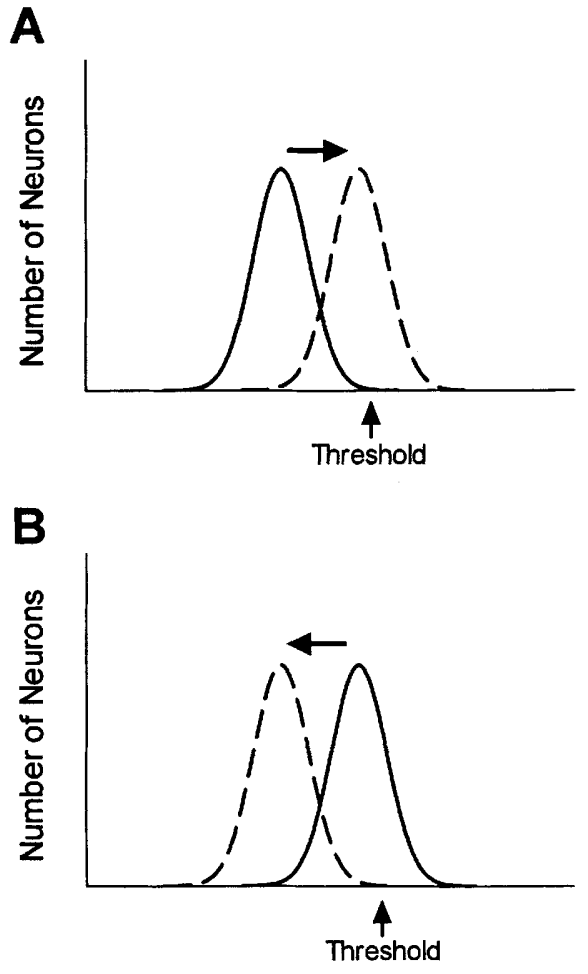

Postsynaptic Depolarization

Figure 1: Abstract, graphical depiction of the revised CA1 decoder hypothesis. A illustrates our previously published LTP experiment (Levy et al. 1995), in which the Schaffer (Sch) collateral input to stratum radiatum is activated at an intensity and frequency that is subthreshold for LTP. In the presence of bicuculline, properly timed, paired activation of the perforant path (PP) and Schaffer collateral inputs shifts postsynaptic excitation sufficiently to exceed the threshold for LTP in many neurons. Thus, an associative interaction between these two laminated afferent systems was demonstrated. $B$ depicts the experiment reported here. Initially the Schaffer collaterals are activated at an intensity and frequency sufficient to induce LTP of the activated synapses. Paired activation of the PP input to stratum moleculare and of the Sch collaterals produces a net inhibition in the CA1 pyramids. The net postsynaptic excitation of the CA1 pyramids is shifted to the left of threshold; as a result, the activated Schaffer collateral synapses do not potentiate. Note how the two experiments are, in a sense, the mirror images of each other.

tion of the stratum moleculare input to CA1 can block the induction of LTP of the CA3-CA1 synapses. If a set of pyramidal cells is slightly past threshold for associative LTP, can an entorhinallike input move these neurons away from the threshold for LTP induction?

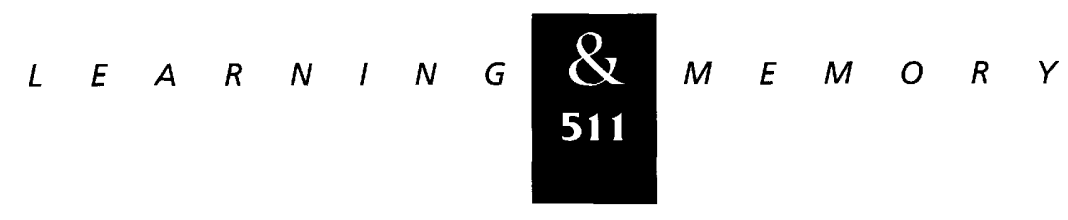


Levy et al.

Some of these data have appeared in abstract form (W.B. Levy, N.L. Desmond, and D.X. Zhang, unpubl.).

\section{Materials and Methods}

The electrophysiological experiments used hippocampal slices from adult, male rats (Hilltop Labs, Sprague-Dawley, 140-225 grams) anesthetized with sodium pentobarbital $(50 \mathrm{mg} / \mathrm{kg}$ i.p.) before decapitation. The preparation of the hippocampal slices was described previously (Colbert and Levy 1992). Briefly, 400- $\mu \mathrm{m}$ transverse slices were maintained in an interface recording chamber at $33^{\circ} \mathrm{C}$. Area $\mathrm{CA} 3$, the dentate gyrus, and subiculum were dissected from the slice to simplify interpretation of the extracellular field potentials. These minislices were equilibrated for at least 60 min before beginning an experiment, certainly long enough for the water-soluble anesthetic to wash out. The artificial cerebrospinal fluid (ACSF) contained $127 \mathrm{~mm} \mathrm{NaCl}, 2 \mathrm{~mm} \mathrm{KCl}, 2 \mathrm{mM} \mathrm{CaCl}_{2}, 1.5$ $\mathrm{mm} \mathrm{MgSO}_{4}, 26 \mathrm{~mm} \mathrm{NaHCO}{ }_{3}, 1.1 \mathrm{~mm} \mathrm{KH}_{2} \mathrm{PO}_{4}$, and 10 mM D-glucose, was bubbled with $95 \% \mathrm{O}_{2}$ and $5 \%$ $\mathrm{CO}_{2}$, and flowed at $1.5 \mathrm{ml} / \mathrm{min}$.

There were two stimulating electrodes and two recording pipettes in each slice. The bipolar stimulating electrodes (twisted pairs of $25-\mu \mathrm{m}$ diam. nichrome wires) were placed in CA1 stratum moleculare and stratum radiatum. Electrical stimulation consisted of $100-\mu \mathrm{sec}$, constant current pulses $(\leqslant 100 \mu \mathrm{A}$ for Schaffer collateral stimulation and $\leqslant 300 \mu \mathrm{A}$ for perforant path stimulation). At the beginning of each experiment, the Schaffer collateral stimulating current was set to evoke a population excitatory postsynaptic potential (pEPSP) that was $-50 \%$ of the maximum response without a marked population spike. The perforant path stimulating current was chosen to prevent any detectable translaminar overlap between the Schaffer collateral and perforant path inputs.

Recording microelectrodes ( $\sim 5 \mathrm{M} \Omega$ ), which were filled with ACSF, were placed in CA1 stratum radiatum and stratum moleculare to record pEPSPs as described previously (Colbert and Levy 1992). Perforant path stimulation evoked a negative-going pEPSP in stratum moleculare and a positive-going pEPSP in distal stratum radiatum. The polarity of the evoked potentials was reversed in response to Schaffer collateral stimulation.

The experimental paradigm consisted of four test periods and two conditioning periods. An ini- tial baseline test period ( $20 \mathrm{~min}$ ) preceded the first conditioning period that was followed by a $30-\mathrm{min}$ test period. A new baseline test period $(20 \mathrm{~min})$ was followed by the second conditioning period and a final test period (30 $\mathrm{min})$.

Conditioning stimulation was always at the test stimulus intensity and consisted of 10 stimulus trains $(1 \mathrm{train} / 200 \mathrm{msec})$ at $100 \mathrm{~Hz}$. There were 5 pulses in each Schaffer (Sch) conditioning train and either 5 pulses (PP) or 10 pulses [PP(Long)] in each perforant path conditioning train. Each experiment used between one and three slices from a given rat. Each slice was used for only one of four different first conditioning paradigms: (1) Sch alone, (2) PP(Long) alone, (3) Sch+PP, or (4) Sch+PP(Long). In all cases, the second conditioning period consisted only of Schaffer conditioning trains.

In one set of experiments, the temporal arrangement of the 5-pulse Schaffer conditioning train and the 10-pulse perforant path train [PP(long)] was varied during the first conditioning period. Assuming that layer 2 and 3 cells in the entorhinal cortex fire synchronously, then direct entorhinal activation of CA1 pyramidal cells would temporally precede their indirect activation via the Schaffer collaterals. In the first group, the trains started together. In the second group, they ended together. In the third group, the Schaffer train occurred in the middle of the perforant path train (i.e., starting with pulse 3 and ending with pulse 7).

The stimulus intensity was often decreased after the first conditioning period and the subsequent test period because LTP induction depends on postsynaptic excitation (McNaughton et al. 1978; Levy and Steward 1979; Kelso et al. 1986; Gustafsson et al. 1987). This adjustment allowed us to compare equivalent responses across different conditioning paradigms. Because only Schaffer conditioning occurred during the second conditioning period (following paired conditioning of the Schaffer collateral and perforant path inputs), the stimulus intensity adjustment biased the experimental outcome against our hypothesis.

The data reported here are measurements of the initial slope of negative-going pEPSPs in stratum radiatum. Mean pEPSP slopes were computed from the individual measurements for 10 -min increments of each test period. All slope values (mean \pm s.E.M.) were normalized across experiments by setting each mean preconditioning baseline response to $100 \%$. The difference between the final 10-min preconditioning average and the final

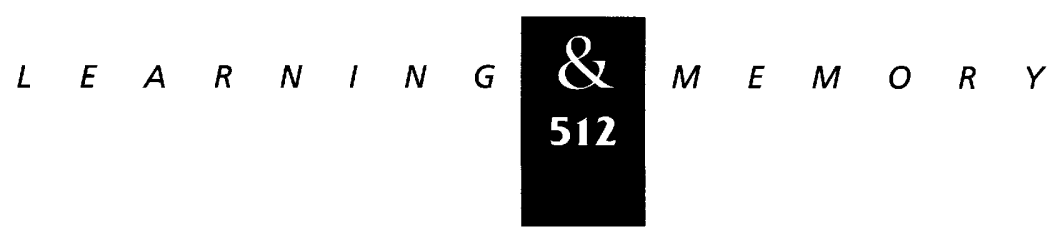


10-min postconditioning average was statistically evaluated across slices using inherently matched, two-tailed Student's $t$-tests. For comparison between groups, one-way analysis of variance was used. $P<0.05$ was required for statistical significance in all cases.

\section{Results}

\section{COMPUTATIONAL ESTIMATE OF EXCITATION} IN CA1 STRATUM RADIATUM

Using recent estimates of CA3-CA1 connectivities and of neuronal activity levels in a behavioral situation that activates the hippocampus, we first estimate the excitation induced in CA1 by CA3. These estimates show that the revised model of CA1 recodings is plausible. Note, however, that we do not imply that these estimates are exact, just reasonable based on the best values available.

Assume that the threshold for LTP is near that for cell firing. We use the following six approximations from the recent literature:

1. There are $2.4 \times 10^{4} \mathrm{CA} 3$ synapses on a CA1 pyramidal cell (Bannister and Larkman 1995; Trommald et al. 1995).

2. For both CA3 and CA1, $12 \%$ of the pyramidal cells are active and are place cells in a test arena (Thompson and Best 1989).

3 . For these $12 \%$ of pyramidal cells, average activity (i.e., the average activity of a place cell in its place field) is $\sim 8.8 \mathrm{~Hz}$ (Thompson and Best 1989).

4. The fraction of the arena covered by one place cell is $\sim 10^{-1}$ (O'Keefe and Burgess 1996).

5. The integrative time constant of a CA1 pyramid is $\sim 10^{-2}$ sec (C.M. Colbert, pers. comm.).

6. If we assume that half of the inputs fail (Andersen et al. 1994), the number of coactive excitatory inputs needed to fire a CA1 pyramid in this 10 -msec interval is $\sim 40$.

These values are correct within better than an order of magnitude. We now use these estimates to assess the plausibility of the hypothesis that many CA1 pyramids are just above or just below the threshold for cell firing.

The probability that a CA3 neuron fires (i.e., is active during a 10-msec window when the rat is in a place field) equals the frequency of $\mathrm{CA} 3$ cell firing multiplied by the duration of this time window multiplied by the fraction of cells firing in the arena multiplied by the place field fraction of a cell in the arena. This probability equals $(8.8)\left(10^{-2}\right)(1.2 \times$ $\left.10^{-1}\right)\left(10^{-1}\right)$ or $-1.06 \times 10^{-3}$ and estimates the active fraction of CA3 neurons during any $10-\mathrm{msec}$ interval.

For computational convenience, we assume random connectivity from CA3 to CA1. The distribution of excitation of CA1 neurons then is binomial $\left(N=2.4 \times 10^{4} ; P=1.06 \times 10^{-3}\right)$ and is wellapproximated by a Poisson distribution with a mean of 25.3. Now, for this Poisson distribution, consider the fraction of CA1 neurons that fire by examining the area in the right-hand tail delimited by the threshold for activation. Using this Poisson distribution and a threshold of 40 active inputs, $4.2 \times 10^{-3}$ of the CA1 neurons will fire every 10 msec. Compared with the estimated firing level of $1.06 \times 10^{-3}$, this value is fourfold too high. That is, the CA1 system could easily be overexcited by its CA3 inputs. Inhibition is thus needed to maintain an appropriate firing rate in CA1 and may play a strong role in selecting which CA1 neurons sustain CA3-CA1 synaptic potentiation.

\section{ELECTROPHYSIOLOGICAL EVIDENCE FOR PERFORANT PATH MODULATION OF CA3-CA1 LTP}

In this section we address the experimental question posed in the Introduction: Can perforant path activation prevent the induction of LTP at the Schaffer collateral-CA1 synapses?

As expected, conditioning the Schaffer collateral input alone significantly potentiated the CA1 stratum radiatum pEPSP $(35.4 \pm 7.2 \% ; t=4.92$, $N=11, P<0.05)$. Subsequent conditioning of the same input (after adjusting the stimulus intensity to obtain a response equivalent in size to that before the first conditioning period) further potentiated the Schaffer collateral-CA1 pEPSP $(9.7 \pm 3.8 \%$; $t=2.55, N=9, P<0.05$ ).

In contrast, simultaneous conditioning of the Schaffer collateral and perforant path inputs in another set of slices elicited significantly less LTP of the Schaffer collateral-CA1 pEPSP than did conditioning of the Schaffer collaterals alone (Fig. 2). On average, this paired conditioning increased the Schaffer collateral-CA1 pEPSP only $16.7 \pm 3.8 \%$ $(t=4.39, N=10)$. The magnitude of the LTP induced by Schaffer collateral conditioning alone differed significantly from that induced by paired conditioning of the Schaffer collateral and perforant path inputs $(F=5.02, d f=1,19, P<0.05)$. After

$$
\text { n....... 圈 }
$$


Levy et al.

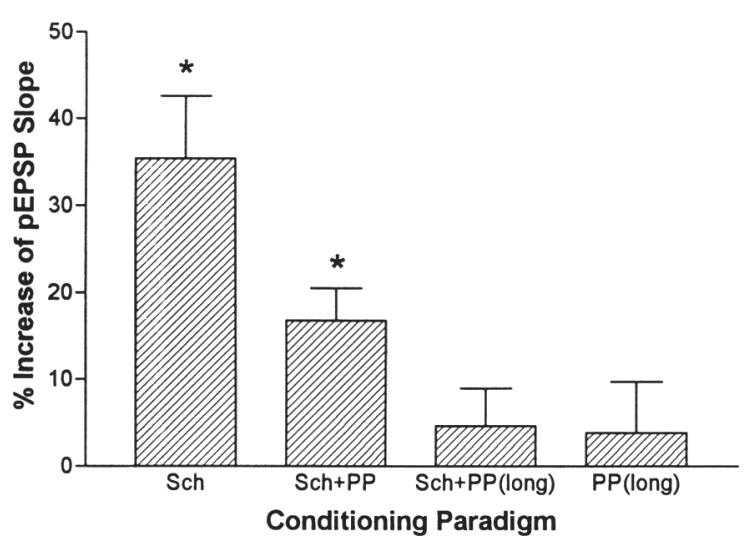

Figure 2: Changes in the normalized Schaffer collateral pEPSP evoked by four different conditioning paradigms. Conditioning the Schaffer (Sch) collateral input alone induces robust LTP. The potentiation induced by simultaneous conditioning of the Schaffer collateral and perforant path (PP) inputs ( $\mathrm{Sch}+\mathrm{PP}$ ) is much smaller than that evoked by Sch conditioning alone. Conditioning either Sch+PP(Long) or the perforant path alone [PP(Long)] does not induce any significant LTP. Values are the mean (+S.E.M.) percent change in the pEPSP slope at $30 \mathrm{~min}$ postconditioning; asterisk $\left(^{*}\right)$ indicates a statistically significant change.

paired conditioning, conditioning the Schaffer collateral input alone did not significantly increase $(4.4 \pm 5.1 \%)$ the Schaffer collateral-CA1 pEPSP slope. At least in part, this small potentiation may reflect the stimulus current reduction made between the two conditioning periods in 8 of $10 \mathrm{ex}$ periments (see Materials and Methods).

When the first conditioning period consisted of 10-pulse perforant path trains that temporally overlapped with the 5-pulse Schaffer collateral trains [Sch+PP(long)], LTP of the Schaffer collateral-CA1 pEPSP was completely prevented (Fig. 2). On average, the pEPSP slope increased only $4.6 \pm 4.3 \%(N=15, P>0.05)$. Figure 3 illustrates the results from one representative experiment. Subsequent conditioning of the Schaffer collateral input alone (with the stimulus intensity adjusted downward for equivalent responses in 5 of 13 slices) significantly potentiated the Schaffer collateral-CA1 pEPSP $(13.0 \pm 3.7 \%, t=3.51, N=15$, $P<0.05$; see Fig. 3 ).

These results contrast with the effect of paired conditioning using synchronous five-pulse PP trains and five-pulse Schaffer collateral trains (see above). The reduced or absent LTP of the Schaffer collateral-CA1 response after paired conditioning can be attributed specifically to modulation by the perforant path input to CA1 rather than to an inability of those particular Schaffer collateral-CA1 pEPSPs to show LTP. Thus, by virtue of comparisons both between slices and within individual slices, perforant path coconditioning effectively suppresses LTP of the Schaffer collateral-CA1 response.

Conditioning the perforant path input alone did not alter the Schaffer collateral-CA1 pEPSP. Ten-pulse PP conditioning trains increased the Schaffer collateral-CA1 pEPSP only $3.8 \pm 5.9 \%$ (Fig. 2). Subsequent conditioning of the Schaffer collateral input alone significantly potentiated the Schaffer collateral-CA1 pEPSP $(28.6 \pm 10.6 \%, t=2.70$, $N=8, P<0.05$ ).

The timing of the 10-pulse perforant path con-
Figure 3: Simultaneous conditioning of the Schaffer collateral and perforant path inputs [Sch+PP(Long)] induces no lasting potentiation; subsequent conditioning of the Schaffer collateral input alone induces robust LTP. Scatterplot of successive normalized Schaffer collateral response slopes. (Insets) Superimposed, average pEPSPs $10 \mathrm{~min}$ before and $30 \mathrm{~min}$ after the indicated conditioning stimulation. The arrow in each inset indicates the mean response postconditioning. Calibration, $0.5 \mathrm{mV}, 5 \mathrm{msec}$.

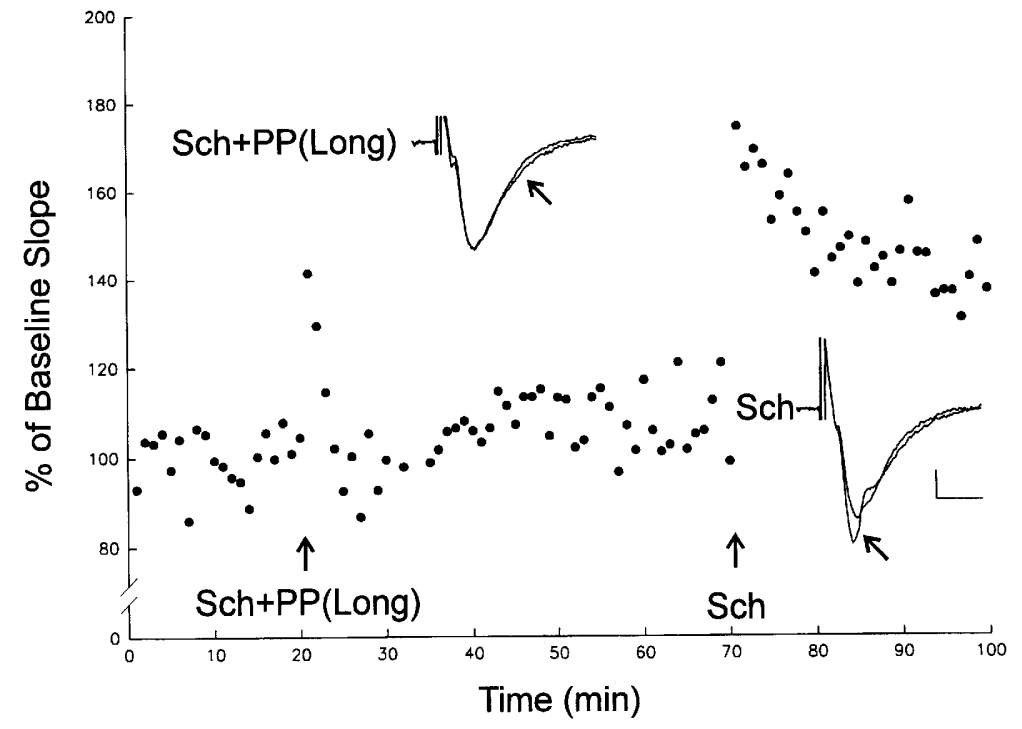

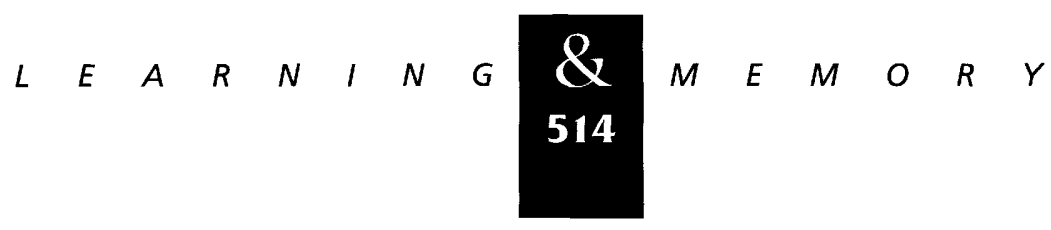


ditioning trains relative to the 5-pulse Schaffer collateral trains influenced the ability of perforant path activation to prevent Schaffer collateral-CA1 LTP (Fig. 4). Perforant path conditioning most effectively prevented LTP of the Schaffer collateralCA1 pEPSP when the perforant path conditioning train slightly preceded $(20 \mathrm{msec})$ and, to some extent, followed $(30 \mathrm{msec})$ each Schaffer collateral conditioning train. On the other hand, when each Schaffer collateral train was delayed to coincide with the end of each perforant path conditioning train, perforant path activation was ineffective at preventing the induction of LTP. In this case, there was a significant increase in the Schaffer collateralCA1 pEPSP (see Fig. $4 ; F=5.68, d f=2,22, P<$ 0.05).

\section{Discussion}

This report has documented that appropriately timed and sufficiently repetitive activation of the perforant path can suppress LTP induced by highfrequency stimulation of the Schaffer collaterals in isolated CA1 slices. This result supports the idea depicted in Figure 1B. In the following discussion, we consider the implications of this and our previous electrophysiological result (illustrated in Fig. 1A) that together suggest that perforant path in-

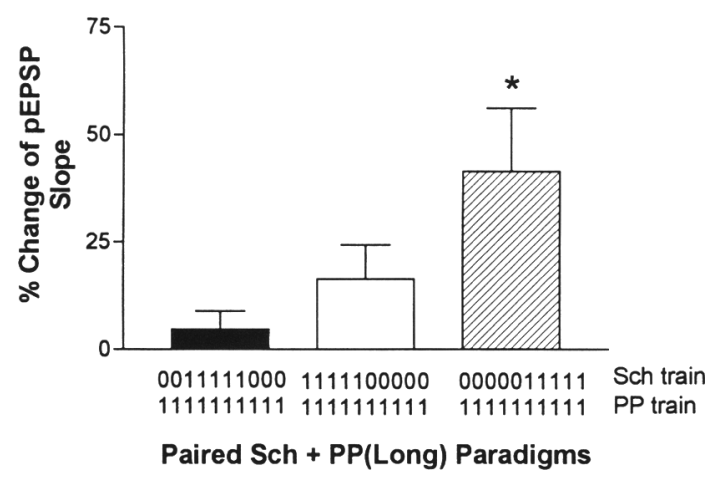

Figure 4: The timing of the Schaffer (Sch) collateral and perforant path (PP) trains influences the extent to which perforant path activation suppresses the induction of Schaffer collateral-CA1 LTP. LTP is suppressed the most when PP conditioning both precedes and follows the Schaffer conditioning. The data shown here are the mean (+S.E.M.) percent changes in the slope of the normalized Schaffer collateral-CA1 response quantified 30 min postconditioning. Each perforant path train consisted of 10 stimuli [PP(Long)]; each Schaffer collateral train had 5 stimuli that overlapped either the middle, beginning, or end of each perforant path train. (0) No stimulus; (1) a stimulus. puts to CA1 bidirectionally influence pyramidal cell firing.

\section{EVOKED INHIBITION AND THE PERFORANT PATH}

Based on anatomical observations (Witter et al. 1988; Desmond et al. 1994), the predominant input to CA1 stratum moleculare travels via the perforant path, is excitatory, and originates from layer 3 neurons of the entorhinal cortex. The morphologically defined excitatory function of this input contrasts with electrophysiological observations using various methods and preparations [field potentials in the isolated CA1 slice (Colbert and Levy 1992), and intracellular recordings in the intact animal (Soltesz 1995), the combined EC-hippocampal slice (Empson and Heinemann 1995), and the isolated CA1 slice (Levy et al. 1995)]. As shown in these studies, perforant path activation generates both inhibition and excitation in CA1.

Here, we have shown that paired activation of the Schaffer collateral and perforant path inputs to CA1 blocks LTP of the Schaffer collateral-CA1 synapses. The voltage-dependent nature of NMDA receptor activation makes associative LTP of Schaffer collateral-CA1 synapses susceptible to inhibitory suppression (see, e.g., Douglas et al. 1982; Holmes and Levy 1997). Because intracellular recordings indicate that perforant path activation creates inhibition in most CA1 pyramids (Lacaille and Schwartzkroin 1988; Lambert et al. 1991; Empson and Heinemann 1995; Levy et al. 1995), it is reasonable to hypothesize that this inhibition is what blocks LTP. Furthermore, based on the dendritic and axonal arbors of interneurons with synapses in CA1 (for review, see Freund and Buzsaki 1996), it seems likely that the inhibition produced by perforant path excitation is relatively nonspecific in locus and spreads throughout the CA1 pyramidal axis including stratum moleculare.

The timing between inhibition and excitation that is optimal for blocking Schaffer collateral-CA1 LTP requires inhibition both to precede and to follow the stratum radiatum excitation. Although the early evoked inhibition would necessarily originate outside the hippocampus in our model, prolonged firing of interneurons in the functioning hippocampus (Fox and Ranck 1975) might provide the later inhibition that our results suggest is important.

\section{CALCULATED POSTSYNAPTIC EXCITATION}

Our overall motivation has been to qualify the

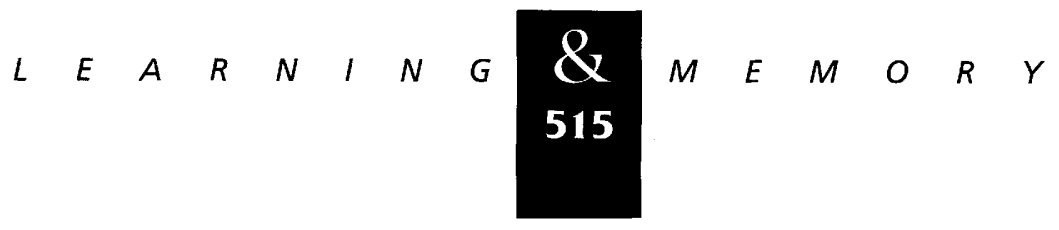


Levy et al.

widely postulated EC-CA1 interaction in which the EC input to CA1 stratum moleculare controls Schaffer collateral-CA1 LTP. Part of this qualification involves estimating the probability distribution of CA3-induced excitation in CA1. The plausibility of the calculated distribution of postsynaptic excitation is crucial to our model and to models used by others.

In creating minimal, biologically realistic models, personal choice and artistry determine which parameters one attempts to satisfy. In our opinion, three fundamental parameters are (1) the number of neurons, (2) the amount of connectivity, and (3) the average firing rate of the neurons. Because computational models that use local associative synaptic modification can not yet achieve realistic numbers, we must rely on estimates from calculations that involve minimal and, at the very least, feasible assumptions.

Using one of many possible sets of reasonable parameters, our calculation produces a model of CA3-induced CA1 excitation in which weak inhibition or weak excitation from the EC selectively determines which CA3-CA1 synapses are potentiated. Although no unique set of parametric values is exclusively supported by this calculation, the calculation does have a self-checking aspect. Specifically, because CA1 and CA3 cell firing rates are quite similar (Thompson and Best 1989), the calculated relative activity level of CA1 should be near the activity level of CA3 itself. This is, in fact, what was found.

The calculation of CA3-induced CA1 excitation is also interesting in its own right. First, it illustrates how far we have come in obtaining the necessary quantified synaptic connectivity and neuronal activity levels in behavioral environments; likewise, it illustrates how far we have yet to go. Given the parametric sensitivity of this calculation, it is quite encouraging that the available values reproduce the desired level of excitation to an accuracy better than an order of magnitude. On the other hand, the parametric sensitivity of the calculation indicates that whereas values known to $20 \%-30 \%$ accuracy are probably satisfactory, parameters specified with only twofold accuracy need more precise measurement. More complex models also merit investigation, for example, those in which theta modulates EC inputs to CA1 (Hasselmo 1995).

By combining the calculation of CA3-induced CA1 excitation with the neurophysiological experiments, we can recover the decoding hypothesis by positing that Schaffer collateral excitation at most
CA1 pyramids is near (i.e., for some cells, slightly above and, for others, slightly below) threshold for cell firing and for associative LTP. The perforant path input then determines which cells sustain LTP. Although most cells are pushed below threshold by the widespread inhibition evoked by perforant path activation, a few cells either stay above threshold on their own or are pushed over this threshold by the excitatory perforant path input. In this way, the EC input to CA1 can modulate the modifiability of Schaffer collateral-CA1 synapses to produce the appropriate decoding of CA3 activity.

Although our inhibitory hypothesis might be a viable alternative to Hasselmo's cholinergic hypothesis (e.g., Hasselmo and Schnell 1994; Hasselmo 1995; Hasselmo et al. 1996), a superior model would combine both perspectives. In Hasselmo's view, cholinergic afferents from the medial septum selectively modulate transmission at the Schaffer collateral-CA1 synapses. Our two hypotheses are certainly not mutually exclusive and could even complement or interact with one other. For example, the inhibitory hypothesis becomes more important if acetylcholine disinhibits CA1 and further increases CA1 excitability.

In sum, if we combine the present results with our previous demonstration of a positive associative interaction (Levy et al. 1995) and the topographic, narrowly excitatory nature of the excitatory EC-CA1 input, the EC-CA1 decoding hypothesis can be resurrected. Furthermore, given that inhibition can permit homosynaptic LTD in the adult CA1 region (Yang et al. 1994), the notion of perforant path-induced translaminar inhibition suggests even more - that there might be a translaminar antiassociative LTD. In this case, the perforant path input would provide inhibition permissive for a homosynaptic LTD in stratum radiatum, a suggestion in agreement with our original CA1 decoding hypothesis (Levy et al. 1990) and appearing in our model as the $\Delta \mathrm{w}^{-}$synaptic modification rule.

\section{Acknowledgments}

This work was supported by National Institutes of Health (NIH) grants RO1 NS15488 and KO2 MH00622 to W.B.L. N.L.D. was also supported by $\mathrm{NIH}$ grant RO1 MH50670. We appreciate the helpful comments of Dr. C.M. Colbert on an earlier version of this paper.

The publication costs of this article were defrayed in part by payment of page charges. This article must therefore be hereby marked "advertisement" in accordance with 18 USC section 1734 solely to indicate this fact.

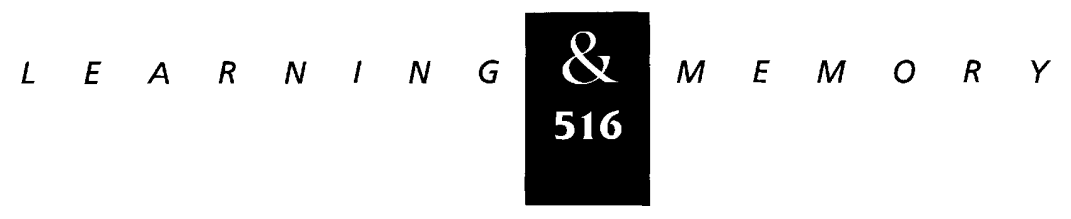




\section{References}

Andersen, P., M. Trommald, and V. Jensen. 1994. Low synaptic convergence of CA3 collaterals on CA1 pyramidal cells suggests few release sites. In Molecular and cellular mechanisms of neurotransmitter release (ed. L. Stjärne, P. Greengard, S. Grillner, T. Hökfelt, and D. Ottoson), pp. 341-351. Raven Press, New York, NY.

Bannister, N.J. and A.U. Larkman. 1995. Dendritic morphology of CA1 pyramidal neurones from the rat hippocampus: II. Spine distributions. J. Comp. Neurol. 360: 161-171.

Burgess, N., M. Recce, and J. O'Keefe. 1994. A model of hippocampal function. Neural Networks 7: 1065-1081.

Colbert, C.M. and W.B. Levy. 1992. Electrophysiological and pharmacological characterization of perforant path synapses in CA1: Mediation by glutamate receptors. J. Neurophysiol. 68: $1-8$.

Desmond, N.L, C.A. Scott, J.A. Jane Jr., and W.B. Levy. 1994. Ultrastructural identification of entorhinal cortical synapses in CA1 stratum lacunosum-moleculare of the rat. Hippocampus 4: 594-600.

Douglas, R.M., G.V. Goddard, and M. Riives. 1982. Inhibitory modulation of long-term potentiation: Evidence for a postsynaptic locus of control. Brain Res. 240: 259-272.

Empson, R.M. and U. Heinemann. 1995. Perforant path connections to area CA1 are predominantly inhibitory in the rat hippocampal-entorhinal cortex combined slice preparation. Hippocampus 5: 104-107.

Fox, S.E. and J.B. Ranck, Jr. 1975. Localization and anatomical identification of theta and complex spike cells in dorsal hippocampal formation of rats. Exp. Neurol. 49: 299-313.

Freund, T.F. and G. Buzsaki. 1996. Interneurons of the hippocampus. Hippocampus 6: 347-470.

Gustafsson, B., H. Wigström, W.C. Abraham, and Y.Y. Huang. 1987. Long-term potentiation in the rat hippocampus using depolarizing current pulses as the conditioning stimulus to single volley synaptic potentials. J. Neurosci. 7: 774-780.

Hasselmo, M.E. 1995. Neuromodulation and cortical function: Modeling the physiological basis of behavior. Behav. Brain Res. 67: 1-27.

Hasselmo, M.E. and E. Schnell. 1994. Laminar selectivity of the cholinergic suppression of synaptic transmission in rat hippocampal region CA1: Computational modeling and brain slice physiology. J. Neurosci. 14: 3898-3914.

Hasselmo, M.E., B. Wyble, and C.E. Stern. 1996. A model of human memory based on the cellular physiology of the hippocampal formation. In Neural networks for neuropsychologists (ed. R. Parks and D. Levine). MIT Press, Cambridge, MA.

Holmes, W.R. and W.B. Levy. 1997. Quantifying the role of inhibition in associative long-term potentiation in dentate granule cells with computational models. J. Neurophysiol. 78: 103-116.

Kelso, S.R., A.H. Ganong, and T.H. Brown. 1986. Hebbian synapses in hippocampus. Proc. Natl. Acad. Sci. 83: $5326-5330$.

Lacaille, J.-C. and P.A. Schwartzkroin. 1988. Stratum lacunosum-moleculare interneurons of hippocampal CA1 region. II. Intrasomatic and intradendritic recordings of local circuit synaptic interactions. J. Neurosci. 8: 1411-1424.

Lambert, N.A., A.M. Borroni, L.M. Grover, and T.J. Teyler. 1991. Hyperpolarizing and depolarizing $\mathrm{GABA}_{\mathrm{A}}$ receptor-mediated dendritic inhibition in area $\mathrm{CA} 1$ of the rat hippocampus. J. Neurophysiol. 66: 1538-1548.

Levy, W.B. 1989. A computational approach to hippocampal function. In Computational models of learning in simple neural systems (ed. R.D. Hawkins and G.H. Bower), pp. 243-305. Academic Press, New York, NY.

Levy, W.B and O. Steward. 1979. Synapses as associative memory elements in the hippocampal formation. Brain Res. 175: 233-245.

Levy, W.B, C.M. Colbert, and N.L. Desmond. 1990. Elemental adaptive processes of neurons and synapses: $\mathrm{A}$ statistical/computational perspective. In Neuroscience and connectionist theory (ed. M.A. Gluck and D.E. Rumelhart), pp. 187-235. Lawrence Erlbaum Assoc., Hillsdale, NJ.

1995. Another network model bites the dust: Entorhinal inputs are no more than weakly excitatory in the hippocampal CA1 region. Hippocampus 5: 137-140.

McNaughton, B.L., R.M. Douglas, and G.V. Goddard. 1978. Synaptic enhancement in fascia dentata: Cooperativity among coactive afferents. Brain Res. 157: 277-293.

O'Keefe, J. and N. Burgess. 1996. Geometric determinants of the place fields of hippocampal neurons. Nature 381: $425-428$.

O'Reilly, R.C. and J.L. McClelland. 1994. Hippocampal conjunctive encoding, storage and recall-avoiding a trade-off. Hippocampus 4: 661-682.

Rolls, E. 1989. The representation and storage of information in neuronal networks in the primate cerebral cortex and hippocampus. In The computing neuron (ed. R. Durbin, C. Miall, and G. Mitchison), pp. 125-159, Addison-Wesley, Wokingham, UK.

Soltesz, I. 1995. A brief history of cortico-hippocampal time with special reference to the direct entorhinal input to CA1. Hippocampus 5: 120-124.

Thompson, L.T. and P.J. Best. 1989. Place cells and silent

$$
\text { n....... 圈 }
$$


Levy et al.

cells in the hippocampus of freely behaving rats. J. Neurosci. 9: 2382-2390.

Trommald, M., V. Jensen, and P. Andersen. 1995. Analysis of dendritic spines in rat CA1 pyramidal cells intracellularly filled with a fluorescent dye. J. Comp. Neurol. 353: 260-274.

Witter, M.P., A.W. Griffioen, B. Jorritsma-Byham, and J.L.M. Krijnen. 1988. Entorhinal projections to the hippocampal CA1 region in the rat: An underestimated pathway. Neurosci. Lett. 85: 193-198.

Yang, X.D., J.A. Connor, and D.S. Faber. 1994. Weak excitation and simultaneous inhibition induce long-term depression in hippocampal CA1 neurons. J. Neurophysiol. 71: 1586-1590.

Received January 26, 1998; accepted in revised form March 18, 1998.

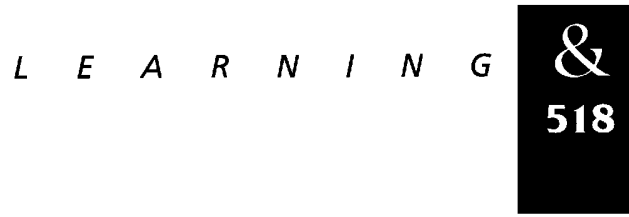




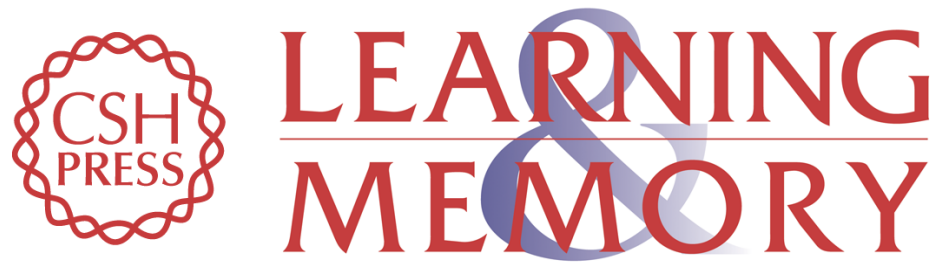

\section{Perforant path activation modulates the induction of long-term potentiation of the schaffer collateral--hippocampal CA1 response: theoretical and experimental analyses.}

W B Levy, N L Desmond and D X Zhang

Learn. Mem. 1998, 4:

Access the most recent version at doi:10.1101//m.4.6.510

References This article cites 25 articles, 5 of which can be accessed free at: http://learnmem.cshlp.org/content/4/6/510.full.html\#ref-list-1

License

Email Alerting

Receive free email alerts when new articles cite this article - sign up in the box at the Service top right corner of the article or click here. 